\title{
The Bone Morphogenetic Protein Roof Plate Chemorepellent Regulates the Rate of Commissural Axonal Growth
}

\author{
Keith D. Phan, ${ }^{1}$ Virginia M. Hazen, ${ }^{1}$ Michele Frendo, ${ }^{1}$ Zhengping Jia, ${ }^{2}$ and Samantha J. Butler ${ }^{1}$ \\ ${ }^{1}$ Neuroscience Graduate Program, Department of Biological Sciences, University of Southern California, Los Angeles, California 90089, and ${ }^{2}$ Hospital for \\ Sick Children, University of Toronto, Toronto, Ontario, Canada M5G 1X8
}

Commissural spinal axons extend away from the roof plate (RP) in response to a chemorepellent mediated by the bone morphogenetic proteins (BMPs). Previous studies have focused on the ability of commissural axons to translate a spatial gradient of BMPs into directional information in vitro. However, a notable feature of this system in vivo is that the gradient of BMPs is thought to act from behind the commissural cell bodies, making it possible for the BMPs to have a continued effect on commissural axons as they grow away from the RP. Here, we demonstrate that BMPs activate the cofilin regulator Lim domain kinase 1 (Limk1) to control the rate of commissural axon extension in the dorsal spinal cord. By modulating Limk1 activity in both rodent and chicken commissural neurons, the rate of axon growth can either be stalled or accelerated. Altering the activation state of Limk1 also influences subsequent guidance decisions: accelerated axons make rostrocaudal projection errors while navigating their intermediate target, the floor plate. These results suggest that guidance cues can specify information about the rate of growth, to ensure that axons reach subsequent signals either at particular times or speeds during development.

\section{Introduction}

During development, axons extend along stereotyped pathways to form precisely ordered neuronal networks (Butler and Tear, 2007). Axons are guided along these pathways by directional information in the embryonic environment, which instructs the axonal growth cones to locally reorganize the actin cytoskeleton and thereby move toward or away from the source of the signal (Dickson, 2002). Additionally, axons must reach directional information at the right time during development and then modulate their speed to navigate the guidance signal. What controls this process? One possibility is that, in addition to "directional" guidance cues that dictate axon orientation, there are also "temporal" guidance signals that regulate the rate of axon outgrowth. A role for temporal cues has been demonstrated recently computationally (Mortimer et al., 2010), but it remains unclear whether this mechanism operates in vivo.

The trajectory of dorsal commissural axons around the circumference of the developing spinal cord is a key model system for elucidating axon guidance mechanisms (Dodd and Jessell, 1988). Commissural neurons (dI1s) arise adjacent to the dorsal

Received Aug. 6, 2010; revised Sept. 3, 2010; accepted Sept. 13, 2010.

This work was supported by Canadian Institutes of Health Research Grant MOP-42396 (Z.J.) and the James H. Zumberge Research and Innovation Fund, March of Dimes Foundation Grant 1-FY07-458, and National Institutes of Health Grant NS 063999 (S.J.B.). We are most grateful to Pico Caroni for the cofilin and Limk1 constructs, Anthony Celeste and John Wozney for the full-length Bmprll construct, Kohei Miyazono for the full-length Bmprlb construct, Jeff Golden for eGFP, Yi Rao for Venus-YFP, Jane Johnson for the Math1::tauGFP reporter mouse line, and Ester Stoekli for Axonin1 antibodies. We also thank Pico Caroni, Ben Novitch, Peter Schieffele, and Ken Yamauchi for helpful discussions, Michael Quick for continued support, and James Briscoe, Artur Kania, and Ben Novitch for comments on this manuscript.

Correspondence should be addressed to Samantha J. Butler, HNB 201, 3641 Watt Way, University of Southern California, Los Angeles, CA 90089. E-mail: butlersj@usc.edu.

DOI:10.1523/JNEUROSCI.4117-10.2010

Copyright $\odot 2010$ the authors $\quad 0270-6474 / 10 / 3015430-11 \$ 15.00 / 0$ midline and are generated by the bone morphogenetic proteins (BMPs) in the roof plate (RP) (Liem et al., 1997). The BMPs subsequently act as a guidance cue to orient commissural axons ventrally (Augsburger et al., 1999). The ability of inductive growth factors, such as the BMPs, to direct remarkably disparate cellular processes during neural development has been observed for other morphogens (Charron and Tessier-Lavigne, 2005; Zou and Lyuksyutova, 2007). However, the mechanisms by which morphogens achieve these distinct functions remain largely unresolved.

Our studies have shown that the repellent activities of the RP BMPs are mediated by the canonical BMP receptors (Bmprs) (Yamauchi et al., 2008), which also transduce the ability of the BMPs to specify dorsal spinal neuronal identity (Wine-Lee et al., 2004). These studies also suggested that commissural neurons remain under the influence of the BMPs as their axons begin to grow ventrally, because outgrowth was disrupted by modulating the activity of BmprIb (Yamauchi et al., 2008). To identify the intracellular effector that specifically transduces the RP chemorepellent in commissural neurons and determine what cellular responses are initiated by BMP guidance cues, we have examined whether a noncanonical intermediate of BMP signaling, Lim domain kinase 1 (Limk1), permits commissural axons to interpret BMP guidance cues in vivo.

Limk1 modulates actin dynamics by regulating cofilin activity (Arber et al., 1998). Limk1/cofilin are downstream effectors of a number of axon guidance cues in vitro (Gehler et al., 2004; Hsieh et al., 2006; Piper et al., 2006; Strochlic et al., 2008; Marsick et al., 2010), including the ability of BMP7 to steer growth cones (Wen et al., 2007). Nonphosphorylated "active" cofilin depolymerizes actin and tends to stimulate neurite outgrowth (Meberg and Bamburg, 2000; Endo et al., 2003). However, polymerization, or 
treadmilling, of actin only occurs when there is a balance between the activation states of Limk1 and cofilin (Sarmiere and Bamburg, 2004). If Limk1 activity is sufficiently elevated, filamentous actin becomes stabilized and growth cone mobility is reduced (Endo et al., 2003; Tursun et al., 2005).

The effect of Limk1/cofilin on axon growth has been clearly demonstrated in vitro, but it remains unclear whether Limk1/ cofilin acts as an intracellular effector of axon guidance in vivo. Here, we show that the BMP-mediated activation of Limk1 in commissural neurons regulates the rate at which they extend axons away from the RP. Intriguingly, when commissural axon growth was accelerated by inactivating the Limk1 pathway, prominent axon guidance errors were observed as they reached the floor plate (FP), a critical intermediate target (Placzek et al., 1990). Thus, the regulation of Limk1/cofilin activity is important for balancing commissural axon growth and guidance decisions and may be critical for the temporal control of commissural axon trajectory.

\section{Materials and Methods}

Immunohistochemistry and in situ hybridization

Antibody staining and in situ hybridization histochemistry was performed on either cryosectioned, dissociated neurons or whole-mount tissues as described previously (Augsburger et al., 1999). Fluorescence and differential interference contrast images were collected on a Carl Zeiss LSM510 confocal and Axiovert 200M and Axioplan 2 microscopes. Images were processed using Adobe Photoshop CS2 and CS4.

The antibodies against the following proteins were used: for mouse: phosphorylated (p)-cofilin at 1:500 (Santa Cruz Biotechnology), neuronal class III $\beta$-tubulin at 1:1000 (Tuj1; Covance), actin at 1:2500 (Millipore), Erm at 1:100 (13H9) (Birgbauer and Solomon, 1989), Tag1 at 1:6 (4D7) (Dodd et al., 1988), green fluorescent protein (GFP) at 1:2000 (3E6; Invitrogen), His at 1:1000 (Covance), and Myc at 1:4 (9E10) (Evan et al., 1985); for rabbit: cofilin at 1:500 (Cytoskeleton Inc.), Limk1 at 1:25 (Cell Signaling Technology), panLh2 (Lhx2/9) at 1:2000 (L1) (Liem et al., 1997), panIsl (Isl1/2) at 1:2000 (K5) (Liem et al., 1997), axonin1 at 1:10,000 (Ruegg et al., 1989), BmprII at 1:100 (Orbigen), and phosphorylated-Smad1/5/8 at 1:1000 (Cell Signaling Technology); for guinea pig: Lhx2 at 1:2000 (Lee et al., 1998) and Lhx9 at 1:500 (Lee et al., 1998). Cyanine 3-, cyanine 5-, or FITC-coupled secondary antibodies were obtained from Jackson ImmunoResearch.

An in situ probe against the $3^{\prime}$ untranslated region of the mouse Limk1 mRNA was prepared using the following primers: forward, $5^{\prime}$-AGGGATCTGAATCCCCAAAC-3', reverse 5'-GAGATTAACCCTCACTAAAGGGAACAATCCCATCCCCCTAAAC- $3^{\prime}$. The underlined region denotes a $\mathrm{T} 3$ polymerase site embedded in the primer sequence. The target sequence was amplified from embryonic day 10.5 (E10.5) mouse spinal cord cDNA by PCR. Qiaquick (Qiagen) purified products were used in an in vitro transcription reaction using the Roche DIG RNA labeling kit.

\section{Generation and analysis of expression constructs}

Math1 enhancer expression constructs were generated as described previously (Yamauchi et al., 2008) by replacing the lacZ reporter gene in the BGZA vector with full-length rat cofilin, a nonphosphorylatable form of rat cofilin, cofilinS3A (Arber et al., 1998), and a truncated form (k1) of mouse Limk1 that is constitutively active (Arber et al., 1998). The BmprII $\Delta$ Lim-GFP and BmprII $\Delta$ Lim-yellow fluorescent protein (YFP) fusion proteins were generated by replacing amino acids 530-1039 encompassing the Lim binding domain in the C terminus of human BmprII (Rosenzweig et al., 1995) with enhanced GFP (eGFP) or Venus-YFP, respectively, as follows: using upstream ( $5^{\prime}$-GCCGCCACATGTCTTCCTCGCTGCAGCGGCC-3') and downstream (5'-GCCGCCACTAGTGACAGGTTGCGTTCATTCTGCA-3') primers, 1-1587 bp of the extracellular domain of BmprII were amplified by PCR using the fulllength receptor as a template. This N-terminal fragment of BmprII was fused to eGFP and inserted into the BGZA vector as above. In ovo elec- troporation was performed as previously described (Yamauchi et al., 2008). Hamburger-Hamilton (HH) stage 15 chicken embryos (AA Laboratory Eggs) were electroporated with a range of concentrations of the Math1 expression plasmids: $0.6-1 \mu \mathrm{g} / \mu \mathrm{l}$ BmprII $\Delta \operatorname{Lim}-\mathrm{GFP}, 0.2 \mu \mathrm{g} / \mu \mathrm{l}$ farnesylated GFP (fGFP), $1.0 \mu \mathrm{g} / \mu \mathrm{l}$ cofilin-myc, $1.0 \mu \mathrm{g} / \mu \mathrm{l}$ cofilinS3A-

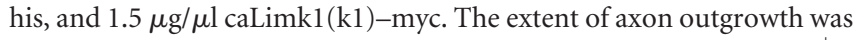
quantified by determining the percentage of GFP-positive $\left(\mathrm{GFP}^{+}\right)$, $\mathrm{Myc}^{+}$, or His ${ }^{+}$commissural axons that had crossed any of four crossing points in their trajectory: mid-dorsal (MD), intermediate (INT), midventral (MV), and FP (see Fig. 2J). At low levels of GFP expression, the trajectories of individual axons can be distinguished easily at the INT, MV, and FP lines. However, the extent of axonal fasciculation at the MD line makes it possible that the MD percentage is an under-representation of the reported result. The number of electroporated commissural neurons was determined by counting the number of $\mathrm{Lhx} 2 / 9^{+}$nuclei with $\mathrm{GFP}^{+}$cell bodies.

Cytomegalovirus (CMV) enhancer expression constructs were generated by inserting a Myc-tagged full-length BmprII, BmprII $\Delta$ Lim-GFP, or BmprII $\Delta$ Lim-YFP into pcDNA3 (Invitrogen). The full-length cofilin/ Limk1 and BmprIb CMV expression vectors were kind gifts from Pico Caroni (Friedrich Miescher Institute, Basel, Switzerland) and Kohei Miyazono (University of Tokyo, Tokyo, Japan), respectively. These constructs were introduced into COS-7 cells using Lipofectamine (Invitrogen) transfection, and the cell was permitted to recover as described previously (Butler and Dodd, 2003). At $24 \mathrm{~h}$ after transfection, the COS cells were harvested in serum-free OptiMEM (Invitrogen), stimulated for $30 \mathrm{~min}$ with $1 \mathrm{ng} / \mathrm{ml}$ recombinant BMP7 protein (R \& D Systems), and dissociated with trypsin-EDTA (Invitrogen). The cell pellets were lysed, run on reducing SDS gels, and transferred to Western blots, which were probed with anti-p-cofilin and anti-actin antibodies (loading control). Western blots were developed with Supersignal West Femto Maximum Sensitivity substrate (Pierce) or ECL Western Blotting Detection Reagents (GE Healthcare), and the net pixel intensity of individual bands measured by densitometry using an Alpha Innotech ChemiImager 4400 .

\section{Generation and analysis of mutant mice}

Limk1 embryos were genotyped by PCR (Meng et al., 2002). To assess the level of phosphorylated cofilin in litters from Limk $1^{+/-}$parents, spinal cords were dissected from E11.5 embryos, lysed, and subjected to a Western blot analysis as above.

\section{Live imaging of electroporated tissue}

$\mathrm{HH}$ stage 15 chicken embryos were electroporated using $0.2 \mu \mathrm{g} / \mu \mathrm{l}$ Math1::fGFP or $0.5 \mu \mathrm{g} / \mu \mathrm{l}$ Math1::BmprII $\Delta$ Lim-GFP and incubated at $37^{\circ} \mathrm{C}$ until they reached either $\mathrm{HH}$ stage 19 or 21 . Without removing the surrounding mesodermal tissue, the spinal cord was dissected into left and right halves, and the nonelectroporated side was discarded. The $\mathrm{GFP}^{+}$half of the spinal cord was mounted lumen side down onto a very thin layer of type I collagen (BD Biosciences) in a glass-bottom tissue culture dish (MatTek), and an additional layer of collagen was added to immobilize the explant. The explant was cultured in a solution of OptiMEM and $1 \times$ penicillin/streptomycin/glutamine (Invitrogen), and the dish was kept at $37^{\circ} \mathrm{C}$ throughout the experiment using either a circulating water bath or a Carl Zeiss heating unit XL S1. Images were taken every 5 min for 6-8 h, with manual refocusing when necessary, using Axiovision software on a Carl Zeiss Axiovert 200M.

\section{Dissociated cell culture}

Rat. For immunohistochemistry, cultures of dissociated E13 rat commissural neurons (Augsburger et al., 1999) were exposed to a $100 \mathrm{ng} / \mathrm{ml}$ solution of BMP7 recombinant protein for $5 \mathrm{~min}$, fixed as described previously (Augsburger et al., 1999), and then labeled. For the Western blot analysis, dorsal halves of E12 rat spinal cords were dissociated using trypsinEDTA (Invitrogen) for $5 \mathrm{~min}$ at $37^{\circ} \mathrm{C}$, and the resulting neurons were plated and cultured overnight at $37^{\circ} \mathrm{C}$ in OptiMEM. These cultures were stimulated by a $6.25 \mathrm{ng} / \mathrm{ml}$ solution of BMP7 recombinant protein diluted in OptiMEM and then analyzed by Western blotting as described above.

Chick. Chick embryos were electroporated at $\mathrm{HH}$ stage 14-15 with either Math1::BmprII $\Delta$ Lim-GFP or CMV::BmprII $\Delta$ Lim-YFP. GFP ${ }^{+}$ electroporated tissue was collected $24-48 \mathrm{~h}$ later ( $\mathrm{HH}$ stage $19-24)$. The embryonic spinal cord was dissected into either a dorsal one-third or an 
intermediate/ventral two-thirds, in L-15 medium (Invitrogen). The neurons within these regions were dissociated using trypsin-EDTA (Invitrogen) for $5 \mathrm{~min}$ at $37^{\circ} \mathrm{C}$. The neurons were resuspended in Ham's F-12 (Invitrogen) medium with L-glutamine, penicillin-streptomycin-glutamine (Invitrogen) and MITO plus Serum Extender (BD Biosciences). The neurons were then plated on UV-treated glass coverslips, immunolabeled, and imaged. The length of the axons and area and perimeter of each growth cone were measured by tracing along or around the Tuj $1^{+}$(axon length) and $\mathrm{Erm}^{+}$(growth cone area and perimeter) labeled regions using NIH Image J.

\section{Generation and analysis of}

whole-mount preparations

Chick embryos were electroporated in ovo at $\mathrm{HH}$ stage 15 and dissected at $\mathrm{HH}$ stage 25 without dispase treatment to remove the spinal cord from the surrounding mesoderm. The resulting explant was cut along the dorsal midline, embedded in collagen, and then immediately fixed and stained with specific antisera. To quantify the number of ipsilaterally projecting GFP ${ }^{+}$ axons, the total number of $\mathrm{GFP}^{+}$axons that had extended to the motor neuron column was scored for the number of $\mathrm{GFP}^{+}$axons that turn ipsilaterally. To quantify the directionality of the contralaterally projecting axons, the total number of $\mathrm{GFP}^{+}$axons present in the FP, both those that had crossed and those in the process of crossing, were scored for the number of $\mathrm{GFP}^{+}$axons that turned either rostrally or caudally. In each case, the number of ipsilaterally or contralaterally projecting axons was expressed as a percentage of the relevant total number of $\mathrm{GFP}^{+}$axons.

\section{Results}

Limk1 is present and active in commissural neurons

Canonical Bmpr signaling can directly ac-

tivate Limk1 in vitro (Lee-Hoeflich et al., 2004; Wen et al., 2007); Limk1 is thought to bind to the tail of the type II Bmpr (BmprII) in a "primed" but inactive state (Foletta et al., 2003; LeeHoeflich et al., 2004) (supplemental Fig. 1A, available at www. jneurosci.org as supplemental material). During BMP binding, Limk1 is phosphorylated and released into the cytosol in an activated form in which it phosphorylates, and thereby inactivates, cofilin (Arber et al., 1998). Previous studies have suggested that Limk1 and cofilin are present ubiquitously in neurons (Sarmiere and Bamburg, 2004). We confirmed that both Limk1 and its binding partner, BmprII, are present in developing commissural neurons as they extend axons. In situ hybridization and immunohistochemistry experiments on E10.5 mouse embryos demonstrated that Limk1 is expressed broadly in postmitotic neurons and their processes in the developing spinal cord (Fig. $1 A-C$ ). In cultures of dissociated rat commissural neurons, Limk1 protein is present throughout the cell body, axon, and growth cone (Fig. $1 D$ ). Strikingly, BmprII is highly enriched in commissural growth cones (Fig. $1 E$, arrowhead).

This localization pattern appears to have functional relevance; first antibodies against p-cofilin label postmitotic neurons in the dorsal and intermediate spinal cord (Fig. $1 F$ ) in a pattern that correlates well with the presence of Limk1 protein (Fig. 1B). p-cofilin is most dramatically expressed in the soma of these neurons, but there is also faint expression in processes (Fig. $1 F$, arrowheads). In particular, Tag1 ${ }^{+}$commissural neurons are labeled (Fig. 1G), suggesting that Limk1 is actively regulating cofilin in these cells. Second, the activation status of cofilin can be regulated in dissociated commissural neurons by the brief application of BMP7 in vitro. In control, unstimulated cultures, p-cofilin was evenly distributed at low levels throughout commissural growth cones (Fig. $2 A, B$ ). In contrast, p-cofilin was upregulated within $5 \mathrm{~min}$ in the BMP7-stimulated growth cones (Fig. 2C,D). Importantly, the overall level of cofilin protein was not significantly different between unstimulated (Fig. 2E) and BMP7-stimulated (Fig. $2 F$ ) cultures, suggesting that BMP signaling results in the rapid phosphorylation of cofilin rather than a redistribution of the protein. Thus, the ability of BMP7 to upregulate p-cofilin temporally precedes BMP7-mediated growth cone collapse, which is observed by 20-30 min (Augsburger et al., 1999), suggesting that collapse could be a biological consequence of cofilin inactivation.

\section{Constitutively activating Limk1 resulted in stalled commissural axon outgrowth}

To determine whether Limk1 can act as an intracellular intermediate translating BMP signaling into the growth of commissural 


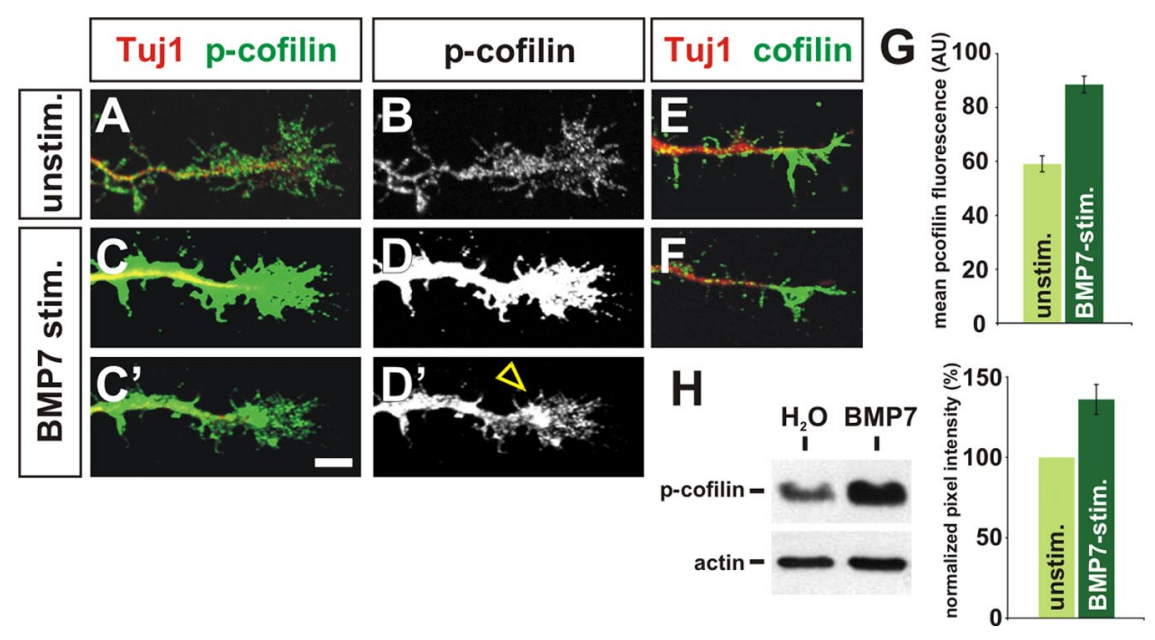

Figure 2. Cofilin is acutely phosphorylated after stimulation with BMP7. A-H, Cultures of dissociated E13 rat commissural neurons were briefly stimulated with either water or BMP7 recombinant protein and then labeled with antibodies against p-cofilin (green, $\boldsymbol{A}-\boldsymbol{D}$ ), total cofilin (green, $\boldsymbol{E}, \boldsymbol{F}$ ), and neuronal class III $\beta$-tubulin (Tuj1; $\boldsymbol{A}, \boldsymbol{C}, \boldsymbol{E}, \boldsymbol{F})$ to reveal neuronal processes (Geisert and Frankfurter, 1989) or subjected to a Western blot analysis $(\boldsymbol{H}) . \boldsymbol{A}, \boldsymbol{B}$, In unstimulated culture conditions, $p$-cofilin is evenly distributed at low levels across commissural growth cones. $C, D$, In contrast, the level of $p$-cofilin was dramatically increased in commissural growth cones treated with BMP7 (open arrowhead), suggesting that the inactivation of cofilin in commissural growth cones is an immediate consequence of BMP7 treatment. $A-D$ were imaged using identical settings on the confocal microscope, whereas $\boldsymbol{C}^{\prime}$ and $\boldsymbol{D}^{\prime}$ were imaged at a lower gain/offset levels to reveal the distribution of $\mathrm{p}$-cofilin in the commissural growth cone after stimulation. $\boldsymbol{E}, \boldsymbol{F}$, The distribution and levels of total cofilin protein is similar in unstimulated $(\boldsymbol{E})$ and BMP7-treated $(\boldsymbol{F})$ commissural growth cones, suggesting that BMP7 treatment directly results in the phosphorylation of cofilin rather than a redistribution of cofilin protein. G, Quantification of mean p-cofilin immunofluorescence in stimulated and unstimulated commissural growth cones. There was a significant increase $\left(p<2.1 \times 10^{-9}\right.$, Student's $t$ test) in the level of $p$-cofilin after addition of BMP7 ( $n=162$ neurons) compared with unstimulated control cultures ( $n=117$ neurons). AU, Arbitrary units. $\boldsymbol{H}$, Western blot of dissociated E13 neurons stimulated with either water or BMP7. There was significant increase $(p<0.0023)$ in the level of $p$-cofilin after BMP stimulation ( $n=4$ experiments). Scale bar: $A-F, 10 \mu \mathrm{m}$.

axons away from the RP, we assessed the effect of modulating Limk1 activity on commissural axon growth in vivo. We first expressed a constitutively active Myc-tagged form of Limk1 (caLimk1-myc) (Arber et al., 1998) within chick commissural axons by in ovo electroporation (Timmer et al., 2001). Constructs were generated containing either fGFP or caLimk1-myc-internal ribosomal entry site (IRES)-fGFP under the control of the Math1 enhancer, which directs the expression of genes to commissural neuron progenitors (Helms et al., 2000). In control experiments, $\mathrm{GFP}^{+}$commissural neurons projected axons normally across the FP by $\mathrm{HH}$ (Hamburger and Hamilton, 1992) stage 23 (Fig. $3 A, B)$. In contrast, commissural neurons electroporated with the Math1::caLimk1-myc-IRES-fGFP construct displayed severe defects in axon extension (Fig. $3 C-E$ ), with $\mathrm{GFP}^{+}$growth cones observed only immediately adjacent to the cell bodies (Fig. 3E, arrowhead). We quantified the extent of outgrowth by determining whether commissural axons had crossed one of four arbitrarily drawn lines in the spinal cord: MD, INT, or MV spinal cord or the FP (Fig. $3 H$ ). By HH stage $23,67 \%$ of control GFP ${ }^{+}$ axons have projected to the intermediate spinal cord, whereas only $8 \%$ of $\mathrm{Myc}^{+}$axons extend this far (Fig. 3I). This defect did not result from an alteration in commissural neural fate, and similar numbers of Lhx $2 / 9^{+}$(Lh2a/b) neurons (Lee et al., 1998) were observed on the electroporated and nonelectroporated sides in both control and experimental conditions (data not shown).

These results suggest that Limk1 activation can inhibit commissural axon outgrowth by phosphorylating cofilin and thereby freezing actin dynamics in the commissural growth cone. Consistent with this model, the stall in axon growth could be rescued by the concomitant electroporation of cofilinS3A-his, a Histagged nonphosphorylatable form of cofilin (Agnew et al., 1995), in commissural neurons (Fig. $3 F, G$ ). Axon outgrowth was partially restored, with $47 \%$ of $\mathrm{His}^{+} \mathrm{Myc}^{+}$axons now reaching the INT line (Fig. 3I). In contrast, the concomitant electroporation of a wild-type form of cofilin did not rescue the caLimk1 phenotype (data not shown).

\section{Lowering Limk1 activity accelerates commissural axon outgrowth}

To assess the effect of lowering Limk1 activity on the commissural axon trajectory, we first analyzed E10.5 Limk1 mutant mice (Meng et al., 2002) using a Math1:: tauGFP reporter (Imondi et al., 2007) to detect the population of commissural axons that arises from Math $1^{+}$neural progenitors. The level of phosphorylated cofilin was decreased in $\operatorname{Limk} 1^{-1-}$ spinal cords (supplemental Fig. S2, available at www.jneurosci.org as supplemental material), suggesting that cofilin is more active in these mice. In the transverse plane of the spinal cord, commissural axons first extend circumferentially away from the dorsal midline (Augsburger et al., 1999) and then project toward the FP at the ventral midline (Tessier-Lavigne et al., 1988; Placzek et al., 1990). The individual trajectories of $\mathrm{GFP}^{+}$axons could be distinguished only at the earliest stages of commissural axon circuit formation. Although the orientation of $\operatorname{Limk} 1^{-/-}$axons away from the RP was indistinguishable from wild-type littermates (Fig. 4A,D), the extent of axon growth in $\operatorname{Limk1}^{-/-}$spinal cords appeared to be more advanced than in the controls (Fig. $4 B, E$, arrowheads). This phenotype could only be unambiguously quantified by examining the number of $\mathrm{GFP}^{+}$commissural axons crossing the FP. At the earliest stages, the spinal cords from the Limk1 ${ }^{-1-}$ embryos had up to $25 \%$ more axons present in the FP compared with wild-type littermates (Fig. $4 C, F, G$ ), suggesting that reducing the level of Limk1 activity accelerates the growth of pioneering Math $1^{+}$commissural axons.

To determine whether BMP signaling regulates the activity of Limk1 in commissural neurons, we sought to decrease the level of Limk1 activity in a BMP-dependent manner in vivo. To this end, we generated a truncated version of BmprII in which the Limk1 binding site on the intracellular tail of BmprII was replaced with GFP (BmprII $\Delta$ Lim-GFP). We predicted that this construct would compete with the endogenous chick BmprII for activation by the RP BMPs, thereby resulting in the sequestration of Limk1 (supplemental Fig. $1 B$, available at www.jneurosci.org as supplemental material). Supporting this model, overexpressing BmprII $\Delta$ Lim-GFP in COS cells resulted in a significant decrease in cofilin phosphorylation (supplemental Fig. $1 C, D$ ), suggesting that the truncated form of BmprII acts to lower Limk1 activity. To assess whether reducing Limk1 activity in a BmprII-dependent manner affects commissural axon outgrowth in vivo, chick embryos were electroporated with either Math1::BmprII $\Delta$ Lim-GFP or Math1::fGFP constructs and permitted to develop until $\mathrm{HH}$ stage 19-21. At these stages, control $\mathrm{GFP}^{+}$axons are in the process of projecting toward the ventral midline; for example, at $\mathrm{HH}$ stage 20 , the Math ${ }^{+}$population of commissural axons have reached the in- 

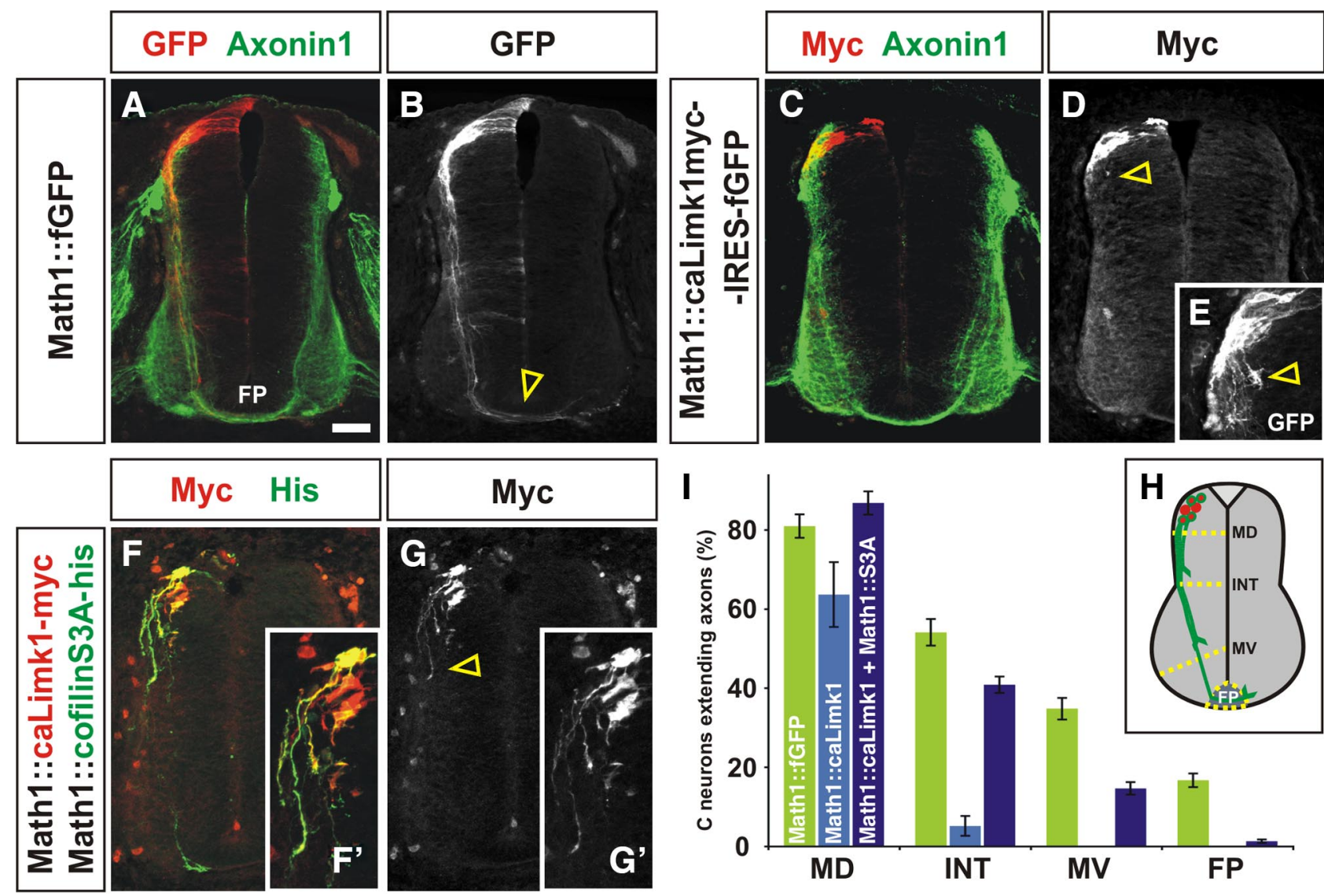

Figure 3. Constitutive activation of Limk1 results in commissural axon outgrowth defects. $\boldsymbol{A}, \boldsymbol{B}$, Chick commissural neurons electroporated with fGFP under the control of the Math1 enhancer (Math1::fGFP) at HH stage 15 extend GFP ${ }^{+}$axons (red) to the FP (arrowhead, $\boldsymbol{B}$ ) by HH stage 24 in concert with the nonelectroporated Axonin $1^{+}$axons (green). $\boldsymbol{C}-\boldsymbol{E}$, In contrast, chick commissural neurons expressing both a Myc-tagged truncated form of Limk1 (caLimk1-myc, red) and GFP have dramatically reduced axon outgrowth at HH stage 24 (arrowhead, D). caLimk1-myc (k1) is constitutively active (Arber et al., 1998); it lacks the Lim domain and thus is neither autoinhibited nor binds to Bmprll. A higher-magnification image $(\boldsymbol{E})$ revealed that the Myc ${ }^{+}$GFP $^{+}$commissural growth cones stall immediately adjacent to the commissural cell bodies (arrowheads, $\boldsymbol{D}, \boldsymbol{E}$ ), suggesting that activation of cofilin has been repressed to such an extent that actin dynamics have been frozen in the commissural growth cone. $\boldsymbol{F}, \boldsymbol{G}$, Consistent with this model, this outgrowth phenotype can be partially rescued by concomitantly electroporating commissural neurons with a nonphosphorylatable form of cofilin, cofilinS3A- his (green). $\boldsymbol{F}^{\prime}$ and $\boldsymbol{G}^{\prime}$ are higher-magnification views of the electroporated region of the spinal cords in $\boldsymbol{F}$ and $\boldsymbol{G}$. Only His ${ }^{+}$(green) or Myc ${ }^{+}$His ${ }^{+}$ (yellow) neurons extend axons into the intermediate and ventral spinal cord (arrows, $\mathbf{G}$ ); very limited axon outgrowth was observed for the Myc ${ }^{+}$(red) neurons. $\boldsymbol{H}$, The extent of the commissural axon outgrowth was quantified by determining whether commissural axons had crossed one of four arbitrary lines in the spinal cord: MD, INT, MV, or FP. Given the potential variation between experiments, only embryos electroporated within the same experiment were compared with each other. $I$, Although $63.7 \pm 8.2 \%$ of commissural neurons expressing Math1::caLimk-myc $(n=24$ sections from 3 embryos) extended a neurite by HH stage 23 , only $8 \%$ reached the INT line ( $p<5.8 \times 10^{-12}$ different from Math1::fGFP control, Student's $t$ test) and none extended to the MV line $\left(p<2.6 \times 10^{-10}\right)$. In contrast, $86.7 \pm 2.9 \%$ of commissural neurons coexpressing Math1::caLimk-myc and Math1::cofilinS3A-his ( $n=108$ sections from 6 embryos) extend axons, of which $47 \%$ reach the INT line $\left(p<1.1 \times 10^{-12}\right.$ different from Math1::calimk-myc alone) and 17\% reach the MV line $\left(p<9.8 \times 10^{-6}\right)$. Scale bar: $\boldsymbol{A}-\boldsymbol{D}, \boldsymbol{F}, \mathbf{G}, 40 \mu \mathrm{m} ; \boldsymbol{E}, \boldsymbol{F}^{\prime}, \boldsymbol{G}^{\prime}, 20 \mu \mathrm{m}$.

termediate spinal cord (Fig. $5 B, F$, arrowheads) but have not yet approached the FP. In striking contrast, commissural axons expressing Math1::BmprII $\Delta$ Lim-GFP have progressed much farther by the same stages, in many cases reaching and crossing the FP (Fig. $5 D, H$, arrowheads). We quantified this behavior for $\mathrm{HH}$ stage 19-21 using the scheme described in Figure $3 H$. At each of these stages, the control and experimental commissural neurons initially extended similar numbers of axons, but in all cases, the BmprII $\Delta$ Lim-GFP ${ }^{+}$axons had projected farther (Fig. 5I). At $\mathrm{HH}$ stage 20 , for example, $<2 \%$ of the control population of axons had reached the FP compared with $>25 \%$ of the experimental population of neurons (Fig. 5I). Accelerated axon growth was only observed when the truncated form of BmprII could be activated by BMPs. Introducing a form of BmprII in which both the extracellular domain (E) and Lim binding domain had been deleted (BmprII $\Delta \mathrm{E} \Delta$ Lim-GFP) into Math1 ${ }^{+}$neurons had no observable effect on the rate of axon growth (Fig. 5J) (supplemental Fig. 3). Thus, the ability of BmprII $\Delta$ Lim-GFP to downregulate Limk1 does depend on the endogenous BMP signals from the RP.
Accelerated commissural axon outgrowth was not a consequence of premature or altered commissural neural development. The activity of canonical effectors of the BMPs, the Smads, was unaffected by the electroporation of either GFP or the experimental constructs (supplemental Fig. 4), and there was no significant difference between the timing and number of Lhx $2 / 9^{+}$cells born in control and experimental spinal cords (supplemental Fig. 5). Rather, the ability of axons to grow faster appears to be a consequence of increased cofilin activity. The overexpression of cofilin in commissural neurons in vivo also resulted in accelerated axon growth to the FP (supplemental Fig. 6). Axons expressing high levels of cofilin grew with very similar kinetics to the axons misexpressing BmprII $\Delta$ Lim-GFP (supplemental Fig. 6G).

Together, these data suggest that elevating cofilin activity, either directly or by reducing Limk1 activation in a BMPdependent manner, results in commissural axons growing faster and thereby reaching their intermediate target $\sim 1 \mathrm{~d}$ earlier than control axons. Strongly supporting this model, imaging live 


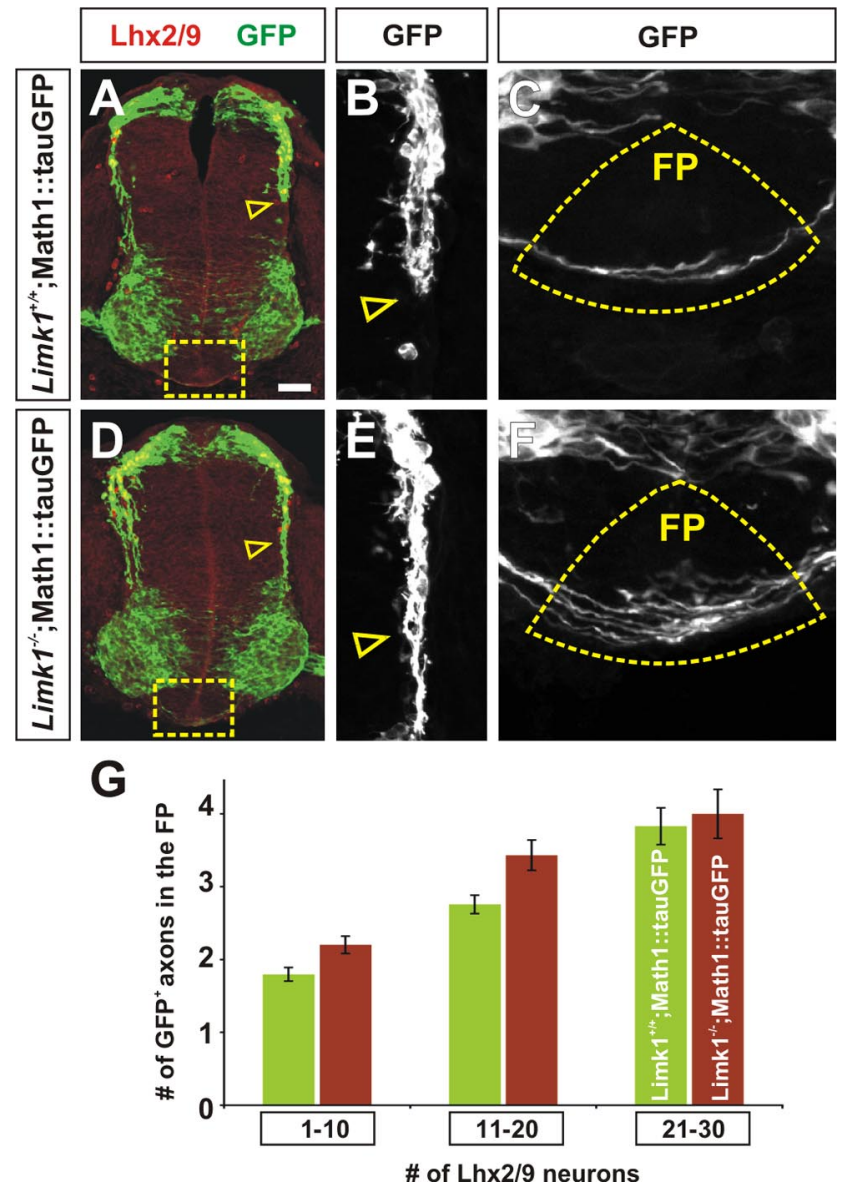

Figure 4. Commissural axons have accelerated trajectories in Limk1 mutant mouse spinal cords. $\boldsymbol{A}-\boldsymbol{F}$, The extent of commissural axon outgrowth was assessed in stage E10.5 wild-type and Limk $1^{-/-}$embryos. Commissural axons were detected using a genetically encoded reporter, Math1::tauGFP (green, $\boldsymbol{A}-\boldsymbol{F}$ ). Sections were also labeled with antibodies against $\mathrm{Lh} \times 2 / 9$ ( $r e d, A, D)$ to normalize the stage of commissural neuron development between sections. $\boldsymbol{A}-\boldsymbol{C}$, In sections of E10.5 wild-type spinal cord with between 11 and $20 \mathrm{Lh} \times 2 / 9^{+}$cells on each side, Math $1^{+}$axons are in the process of projecting through the intermediate spinal cord (arrowhead, $\boldsymbol{A}, \boldsymbol{B}$ ) with relatively few Math $1^{+}$axons having reached the FP. The FP is boxed in $\boldsymbol{A}$ and is shown at higher magnification in $\boldsymbol{C}$. $\boldsymbol{D}-\boldsymbol{F}$, In contrast, in Limk $1^{-/-}$littermates at the same stage of commissural neural development, more axons have projected thought the intermediate spinal cord (arrowhead, $\boldsymbol{D}, \boldsymbol{E}$ ) and have reached the FP. The FP is boxed in $\boldsymbol{D}$ and is shown at higher magnification in $\boldsymbol{F}$. $\boldsymbol{G}$, Quantification of the total number of axons in the FP at three stages of commissural neuronal development: $1-10 \mathrm{Lh} \times 2 / 9^{+}$neurons (wild-type, $n=133$ axons from 16 embryos; Limk1 ${ }^{-/-}, n=109$ axons from 10 embryos), $11-20$ Lhx2/9 ${ }^{+}$neurons (wild-type, $n=99$ axons from 15 embryos; Limk $1^{-/-}, n=51$ axons from 7 embryos), and $21-30 \mathrm{Lhx2} / 9^{+}$neurons (wild-type, $n=47$ axons from 7 embryos; Limk ${ }^{-/-}, n=22$ axons from 4 embryos). For the two earliest stages of development, there are $20-25 \%$ more Math1 ${ }^{+}$axons in the FP in Limk $1^{-/-}$mutants compared with wild-type littermates (1-10 neurons, $p<0.0038$, Student's $t$ test; $11-20$ neurons $p<0.0021)$. At later stages, there is no significant difference ( $p>0.34$ ) in the numbers of axons crossing the FP in wild-type and Limk $1^{-1-}$ embryos. However, the tract becomes increasingly fasciculated in the FP at later stages, making it challenging to distinguish individual axon trajectories. Scale bar: $A, B, D, E, 40$ $\mu \mathrm{m} ; C, F, 5 \mu \mathrm{m}$.

axons in explants of electroporated chick tissue in vitro demonstrated that the BmprII $\Delta$ Lim-GFP ${ }^{+}$axons had a $30 \%$ faster average velocity than control $\mathrm{GFP}^{+}$axons (Fig. $5 \mathrm{~K}$ ) as they grew through the dorsal spinal cord. This increased rate of growth would permit them to grow an average of $100 \mu \mathrm{m}$ farther than control axons in a $24 \mathrm{~h}$ period, a figure strikingly consistent with the changes in axon length observed after electroporating the spinal cord with BmprII $\Delta$ Lim-GFP (Fig. 5I). Interestingly, the growth rate of $\mathrm{GFP}^{+}$and BmprII $\Delta$ Lim-GFP ${ }^{+}$axons was differ- ent only in the dorsal spinal cord; there was no significant difference $(p>0.26)$ between the growth rate of these two populations of axons in the ventral spinal cord (Fig. $5 \mathrm{~K}$ ). The velocity of control GFP ${ }^{+}$axons was $30 \%$ faster in the ventral spinal cord, whereas BmprII $\Delta$ Lim-GFP ${ }^{+}$axons projected through the ventral spinal cord at a rate comparable with that in the dorsal cord (Fig. $5 K$ ). Together, these results demonstrate that Math $1^{+}$commissural axons normally extend more slowly in the dorsal spinal cord than in the ventral spinal cord. Reducing the activity of Limk1 appears to release commissural axons from this inhibition of growth, such that they now grow at a constant "accelerated" speed through the spinal cord.

Lowering Limk1 activity results in long filopodial protrusions Activated cofilin depolymerizes actin filaments and thereby increases the pool of actin monomers needed at the leading edge of a cell for motility (Sarmiere and Bamburg, 2004). Thus, the cellular basis for accelerated commissural axon growth is likely to be an increase in the rate of actin polymerization. We assessed this possibility by determining whether our manipulations of cofilin activity altered the morphology of the actin cytoskeleton in growth cones. After the electroporation of either cofilin or BmprII $\Delta$ Lim-GFP, dorsal spinal neurons were observed to extend growth cones with longer, more complex filopodia both in vivo (Fig. $6 B$ ) and in vitro (Fig. $6 E, F$ ) than control growth cones (Fig. $6 A, C, D$ ). No difference in overall neurite length was observed in vitro (Fig. $6 G$ ), presumably because the neurons are no longer adjacent to a source of BMPs. However, the perimeter of the experimental dissociated growth cones was $40 \%$ longer than that of control growth cones (Fig. 6G). Increased protrusions from the leading edge are consistent with actin polymerization being more dynamic in the growth cone.

Together, these observations support the model that the balance between the activation states of cofilin and Limk1 controls rate of axon outgrowth in vivo by regulating how fast actin treadmills in the growth cone. This mechanism may be a general one: both Limk1 and cofilin are widely expressed in neurons (Sarmiere and Bamburg, 2004) (Fig. 1 A,B), and dissociated intermediate and ventral spinal neurons misexpressing BmprII $\Delta$ Lim-YFP also have more complex growth cones (supplemental Fig. 7).

\section{Accelerated commissural neurons make guidance errors projecting toward and across the FP}

These studies indicate that, in addition to polarizing commissural axons, a key role of the BMP chemorepellent in vivo is to regulate the rate at which commissural axons extend away from the dorsal midline. The existence of such an axon outgrowth-regulating activity would permit commissural axons to reach an intermediate targets, such as the FP, at a particular time in development. We thus assessed whether accelerated axon outgrowth affected the ability of commissural axons to make the correct guidance decisions in the ventral spinal cord.

The trajectory of axons originating from Math ${ }^{+}$neurons was visualized in longitudinal "open book" preparations of the spinal cord (Bovolenta and Dodd, 1990). Control GFP ${ }^{+}$chick axons (Fig. $7 A, B$ ) behaved similarly to what has been described previously for the analogous class of rodent axons (Bovolenta and Dodd, 1990, 1991; Wilson et al., 2008) as follows: by $\mathrm{HH}$ stage 24 , there were two populations of axons, the well described commissural axons that project contralaterally, first across the FP then rostrally toward the brain (Fig. $7 A$, solid bracket; $B$, arrowhead) and another, later-born class of axons that project ipsilaterally, turning rostrally before crossing the 


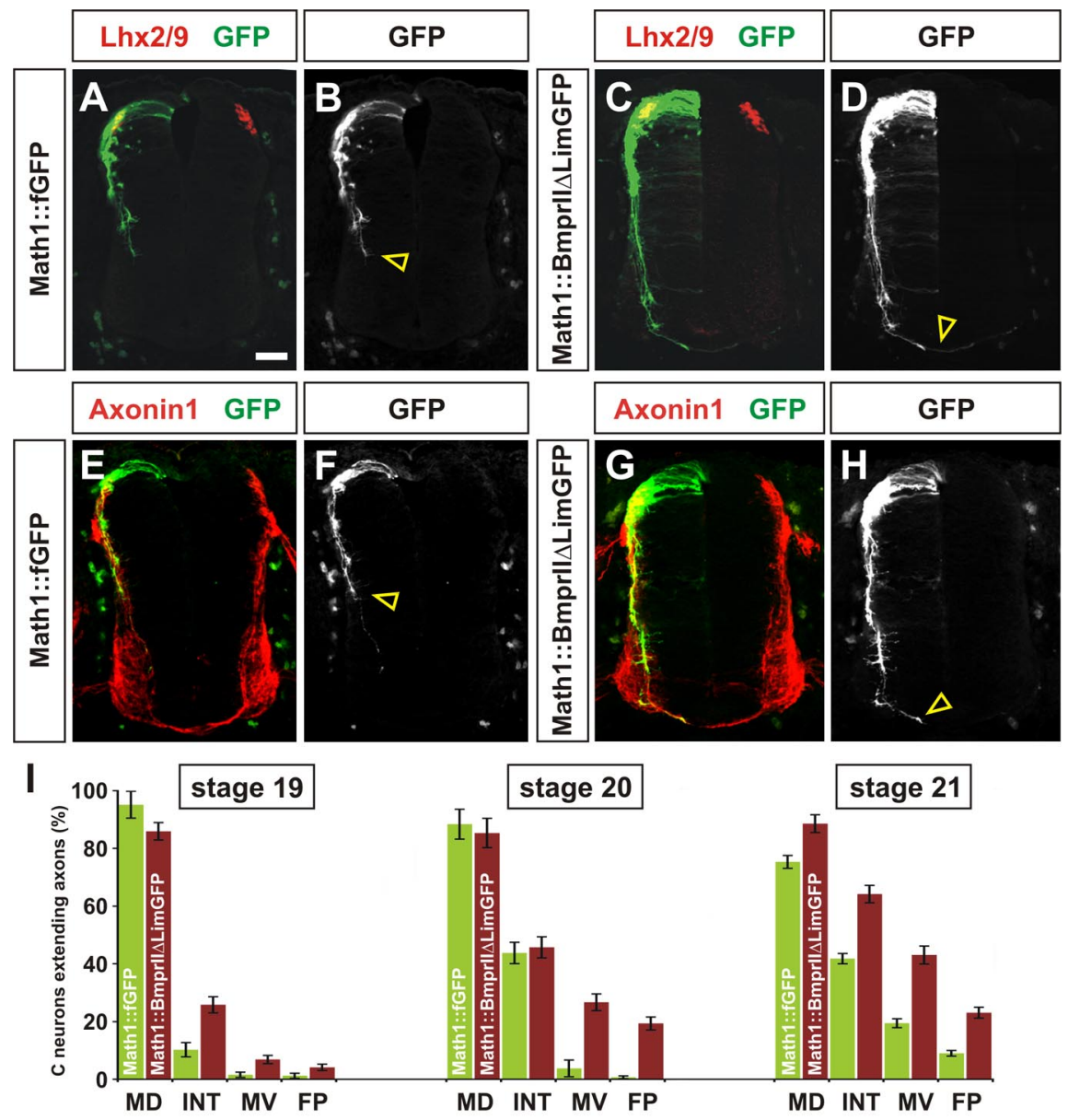

J

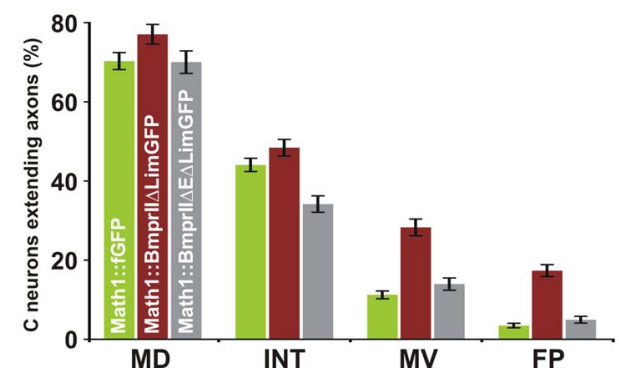

K

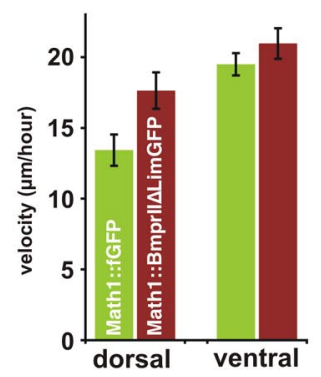

Figure 5. Reducing Limk1 activity by truncating Bmprll results in accelerated axon growth. $\boldsymbol{A}-\boldsymbol{K}$, To assess whether Limk1 functionally interacts with Bmprll in commissural neurons, a truncated Bmprll construct was generated under the control of the Math1 enhancer (green, Math1:: Bmprll $\Delta$ Lim-GFP) in which the Limk1 binding site on the intracellular tail of Bmprll was replaced with GFP. Commissural neurons were labeled with antibodies against Lhx2/9 (red, $\boldsymbol{A}, \boldsymbol{C}$ ) or Axonin1 (red, $\boldsymbol{E}, \mathbf{G}$ ). Commissural neurons electroporated with full-length Bmprll project axons in a similar manner to control GFP ${ }^{+}$axons (data not shown). $A, B, E$, $\boldsymbol{F}$, Chick neurons electroporated with a control Math1::fGFP construct at HH stage 15 have extended GFP ${ }^{+}$axons into the intermediate spinal cord by $\mathrm{HH}$ stage 20. C, D, G, $\boldsymbol{H}$, In contrast, many commissural neurons electroporated with Math1::Bmprll $\Delta$ Lim-GFP have extended axons to the FP (arrowhead, $\boldsymbol{D}, \boldsymbol{H}$ ) by HH stage 20 . $\boldsymbol{I}$, Axon outgrowth was quantified as follows. For HH stage 19, $95 \pm 4.6 \%$ commissural neurons expressing Math1::fGFP extended axons, of which $10 \%$ had projected to the INT line ( $n=90$ sections from 5 embryos). A similar number, $86 \pm 3.0 \%$ ( $p>0.05$, Student's $t$ test) of commissural neurons expressing Math1::Bmprll $\Delta$ Lim-GFP sections had extended axons, but $\sim 30 \%\left(p<1.4 \times 10^{-7}\right.$ ) of these axons had reached INT line ( $n=81$ sections from 5 embryos). For HH stage 20, $88 \pm 5.1 \%$ of commissural neurons expressing Math1::fGFP extended axons, with $4.5 \%$ of these axons projecting to the MV line ( $n=52$ sections from 4 embryos). A similar number, $85 \pm$ $5.1 \%(p>0.33)$ of commissural neurons expressing Math1::Bmprll $\Delta$ Lim-GFP have extended axons, but $>32 \%(p<2.6 \times$ $10^{-11}$ ) of these had reached MV line ( $n=51$ sections from 4 embryos). For HH stage $21,75.3 \pm 2.2 \%$ of commissural neurons expressing Math1::fGFP extended axons, with 12\% of these axons projecting to the FP ( $n=74$ sections from 5 embryos). In this experiment, $88.6 \pm 3.0 \%$ of commissural neurons expressing Math1::Bmprll $\Delta$ Lim-GFP had extended axons, but $>26 \%(p<$ $1.4 \times 10^{-13}$ ) of these had reached FP ( $n=42$ sections from 3 embryos). J, To assess whether endogenous BMP binding was required for accelerated axon growth, a construct lacking both the extracellular domain and Lim binding domain (Math1: Bmprll $\Delta \mathrm{E} \Delta \mathrm{Lim}-\mathrm{GFP}$ ) was electroporated into commissural neurons. By HH stage 21, there was no significant difference between extent of outgrowth of control GFP ${ }^{+}$axons ( $n=114$ sections from 7 embryos) compared with Bmprll $\Delta \mathrm{E} \Delta$ Lim-GFP $^{+}$axons ( $n=97$ sections from 5 embryos) at either the MV $(p>0.057)$ or FP $(p>0.066)$ lines. $\boldsymbol{K}$, The rate of growth of electroporated
FP (Fig. 7A, dotted bracket; $B$, arrow). This subdivision of Math $1^{+}$neurons arises from spatial and temporal segregation of the Lhx9 transcription factor (Lee et al., 1998; Wilson et al., 2008). Lhx9 ${ }^{+}$neurons were born normally after electroporation with the Math1::BmprII $\Delta$ Lim-GFP construct and project in a polarized manner around the circumference of the spinal cord (supplemental Fig. 5). However, their axons displayed guidance errors on reaching the ventral spinal cord. Very few of the accelerated $\mathrm{GFP}^{+}$axons were observed to turn ipsilaterally (Fig. $7 C-E$ ), and, although many of the accelerated commissural axons still projected rostrally, $\sim 10 \%$ of the axons inappropriately turned caudally (Fig. $7 C, D, F$, arrowheads). Very similar guidance errors were observed when Math $1^{+}$axons were electroporated with the cofilin-myc construct (Fig. $7 E, F$ ), suggesting that these errors are a consequence of modulating the status of cofilin activity.

Thus, elevating the activation state of cofilin has a profound consequence for the two populations of Math $1^{+}$axons: they ignore and/or misinterpret guidance signals, such that they make significant turning errors.

\section{Discussion}

The BMP RP chemorepellent controls the rate of commissural axon outgrowth by activating Limk1

In our previous work, we demonstrated that the BMP morphogens unexpectedly had axon guidance activity (Augsburger et al., 1999; Butler and Dodd, 2003) in addition to their ability to specify cell fates (Hogan, 1996). Other groups subsequently showed that this property is a general one for other morphogens, such as Shh (Charron et al., 2003), the Wnts (Lyuksyutova et al., 2003), and the fibroblast growth factors (Irving et al., 2002; Shirasaki et al., 2006). However, the signal transduction pathway(s) by which morphogens signal to

$\leftarrow$

axons was directly determined by imaging live cultures as they extended through either the dorsal (HH stage 19) or ventral (HH stage 21) spinal cord. In the dorsal spinal cord, axons electroporated with Math1::fGFP have a velocity of $13.4 \pm 1.1$ $\mu \mathrm{m} / \mathrm{h}$ ( $n=28$ neurons), whereas axons electroporated with Math1::Bmprll $\Delta$ Lim-GFP grow significantly faster ( $p<$ 0.018 ) with a velocity of $17.6 \pm 1.3 \mu \mathrm{m} / \mathrm{h}$ ( $n=31$ neurons). In contrast, there is no significant difference between the rate of growth $(p<0.26)$ in the ventral spinal cord; the control $\mathrm{GFP}^{+}$axons have an average velocity of $19.5 \pm 0.8 \mu \mathrm{m} / \mathrm{h}$ ( $n=64$ neurons), whereas the Bmprll $\Delta$ Lim-GFP $^{+}$axons have a velocity of $21.0 \pm 1.1 \mu \mathrm{m} / \mathrm{h}$ ( $n=45$ neurons). Scale bar: $\boldsymbol{A}-\boldsymbol{H}, 40 \mu \mathrm{m}$. 


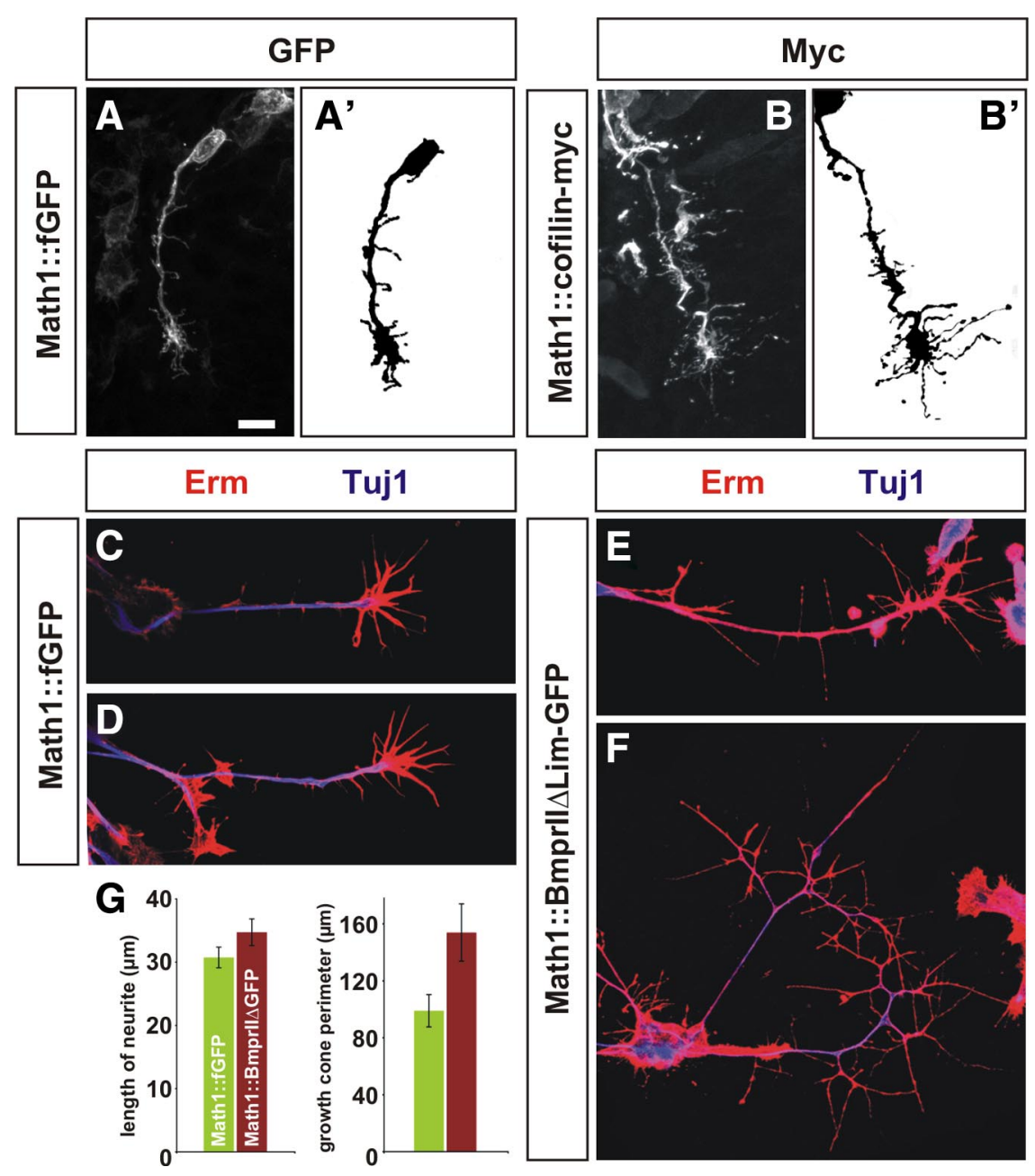

Figure 6. Accelerated commissural neurons have more complex growth cones. $\boldsymbol{A}, \boldsymbol{B}$, Commissural neurons electroporated with either Math1::Bmprll $\Delta$ Lim-GFP (data not shown) or Math1::Cofilin-myc $\left(\boldsymbol{B}, \boldsymbol{B}^{\prime}\right)$ extend growth cones in vivo that appear to have longer, more extensive filopodia that control growth cones $\left(\boldsymbol{A}, \boldsymbol{A}^{\prime}\right) . \boldsymbol{C}-\boldsymbol{F}$, To assess the morphology of electroporated commissural neurons in vitro, chick embryos were electroporated at $\mathrm{HH}$ stage 15 , and cultures of dissociated dorsal neurons were generated at $\mathrm{HH}$ stage 24. Control $(\boldsymbol{A}, \boldsymbol{B})$ and Bmprll $\Delta \operatorname{Lim}^{-G F P}{ }^{+}(\boldsymbol{C}, \boldsymbol{D})$ commissural growth cones were labeled with antibodies against class III $\beta$-tubulin (Tuj1, blue) and the Erm (ezrin, radixin, moesin) complex (red) to reveal the growth cone morphology. The Bmprll $\Delta$ Lim-GFP $^{+}$growth cones are dramatically more complex, with longer and more extensive filopodia. $\boldsymbol{E}$, Quantification revealed that, although there was no significant difference between control and experimental neurons in the length of the longest neurite ( $p>0.13$, Student's t test), the average perimeter of the Bmprll $\Delta$ Lim-GFP ${ }^{+}$growth cones $(150.4 \pm 2.0 \mu \mathrm{m}, n=41)$ is $>50 \%$ larger $(p<0.015)$ than that of the controls $(90.9 \pm 1.1 \mu \mathrm{m}, n=50)$. Scale bar, $10 \mu \mathrm{m}$.

convey guidance information have remained largely unresolved. Here, we link the BMP RP chemorepellent to Limk1/cofilin, intracellular effectors that directly regulate the actin cytoskeleton. Previous studies in vitro have established both that BMP signaling can activate Limk1 (Foletta et al., 2003; Lee-Hoeflich et al., 2004; Wen et al., 2007) and that the activation states of Limk1/cofilin can control axon extension (Meberg and Bamburg, 2000; Endo et al., 2003; Endo et al., 2007). Here, we provide a biological context for these activities: BMPs from the RP increase Limk1 activity in commissural neurons to control the rate at which they extend axons through their transverse trajectory through the spinal cord. The BMPs appear to act locally, limiting the rate of axon extension for the first portion of the commissural trajectory through the dorsal spinal cord. When the activity of Limk1 is reduced, commissural axons grow faster along this region of their dorsal trajectory and therefore reach the FP at an earlier stage in development.

The ability of the BMP RP chemorepellent to control the rate of axon outgrowth in vivo is a novel activity for the BMPs. Previ- ous studies had shown that, like all "classical" guidance signals, the BMPs can provide directional information when presented in front of the growth cone in vitro (Augsburger et al., 1999; Wen et al., 2007). We had previously argued that this result suggests that the BMPs provide a polarizing signal for commissural cell bodies to orient the growth cone away from the dorsal midline. The existence of a polarizing activity remains a possibility, given that loss of BMP signaling disrupts axonal polarity (Butler and Dodd, 2003; Yamauchi et al., 2008). However, the polarizing activity of the BMPs does not appear to be mediated by Limk1, because the polarity of axon outgrowth remained intact after electroporation with caLimk1-myc (Fig. $2 D, E)$, and it appears to be either redundant with other signals or weaker than the outgrowth regulating activity of the BMPs in this context (Butler and Dodd, 2003; Yamauchi et al., 2008; present study).

Given that Limk1/cofilin are widely expressed in neurons and that Limk1/Cofilin are downstream effectors of a number of axon guidance cues in vitro (Gehler et al., 2004; Hsieh et al., 2006; Piper et al., 2006), it seems likely that other guidance cues also modulate the balance between the activation states of Limk1/cofilin to confer "rate-regulating" information to axons. The ability of such cues to control axonal growth rate in vivo has not been reported previously, but the unusual geometry of our system, in which the putative gradient of BMPs is highest behind the neuronal cell bodies, may have facilitated our ability to detect it. Axon guidance studies have primarily focused on identifying the signals and cellular rearrangements that result in oriented axonal growth by presenting directional guidance cues either in front of or lateral to the growth cone. In contrast, the ability of guidance signals to regulate the rate of axon outgrowth may be easier to distinguish when these cues are behind the extending growth cone, as is observed when axons are initiating their trajectory or are extending away from an intermediate target.

\section{The specification of axonal rate information can regulate axon guidance decisions}

A consequence of increasing cofilin activity in commissural neurons is their increased rate of growth in the dorsal portion of their trajectory through the spinal cord. Interestingly, these accelerated axons subsequently make guidance errors on encountering the FP. The mechanistic basis of these errors remains unresolved. One possibility is that they are a nonspecific consequence of the overactivation of cofilin in commissural growth cones. We think this possibility unlikely given that control and experimental axons grow at a comparable speed in the ventral spinal cord (Fig. $5 K$ ), and remarkably similar guidance defects are observed after 

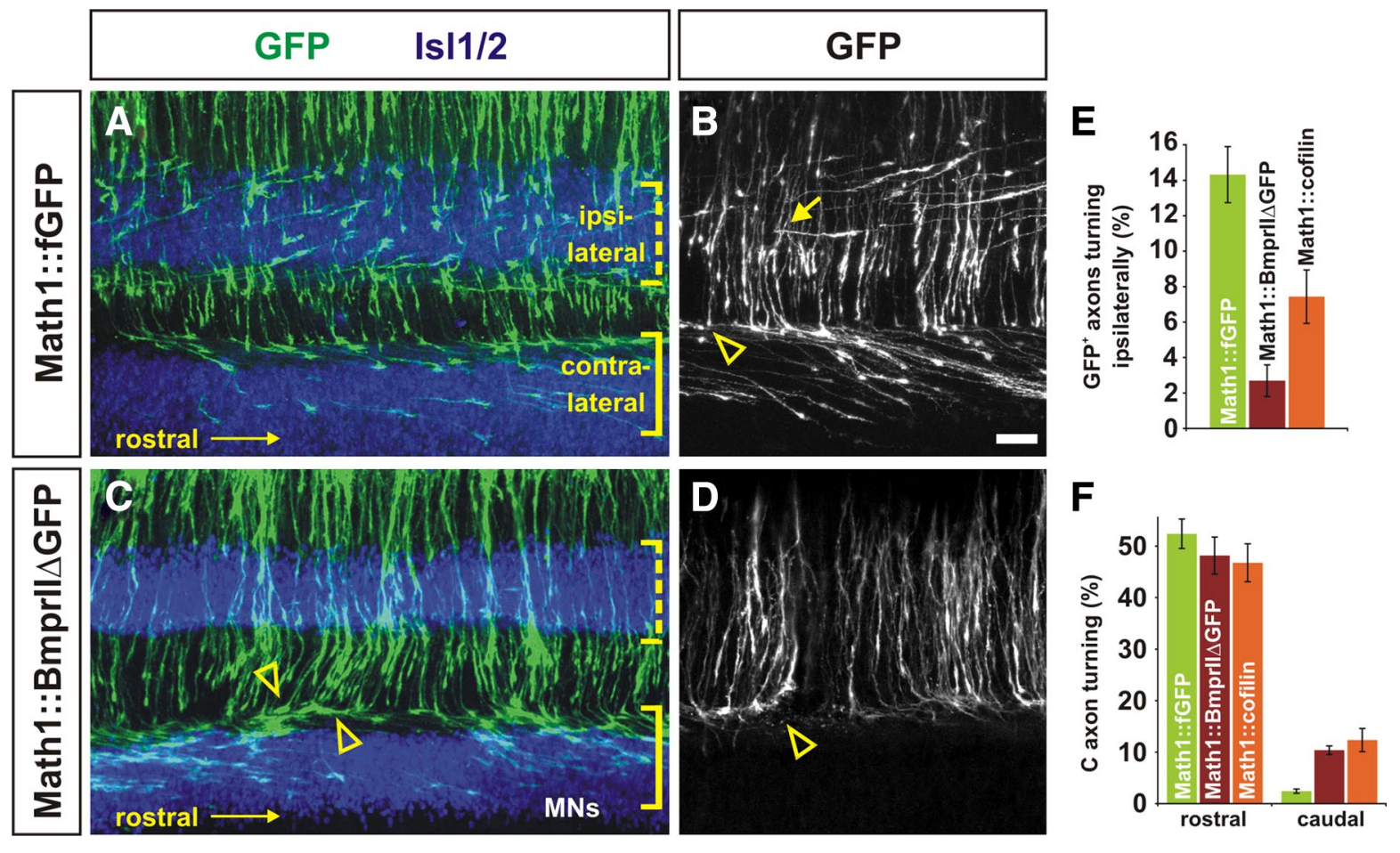

Figure 7. Accelerated commissural axons make guidance errors projecting toward and across the floor plate. $\boldsymbol{A}-\boldsymbol{D}$, To determine the consequence of accelerated axon growth, we examined the behavior of electroporated commissural axons crossing the FP. Chick embryos were electroporated at HH stage 15 , and longitudinal open book preparations of the spinal cord were generated at $\mathrm{HH}$ stage 24. The position of the FP was visualized by labeling the flanking motor neuron column with antibodies against Islet $1 / 2(|\mathrm{~s}| 1 / 2$, blue, $\boldsymbol{F}, \boldsymbol{H})$. The images in $\boldsymbol{A}-\boldsymbol{C}$ are flattened confocal stacks, whereas the image in $\boldsymbol{D}$ is a single confocal slice to facilitate visualizing axons turning rostrocaudally after crossing the FP. $\boldsymbol{A}, \boldsymbol{B}$, By HH stage 24, control neurons electroporated with Math1::fGFP have projected axons ventrally and then sharply rostrally, to project toward the brain. There are two classes of GFP ${ }^{+}$axons, an ipsilateral population that turns before the FP (bracket in $A$, arrow in $\boldsymbol{B}$ ) and a contralateral commissural population that turns after crossing the FP (bracket in $\boldsymbol{A}$, arrowhead in $\boldsymbol{B}$ ). $\boldsymbol{C}, \boldsymbol{D}$, In contrast, by the same stage, neurons electroporated with Math1::Bmprll $\Delta$ Lim-GFP project very few axons ipsilaterally (dotted bracket, $\boldsymbol{C}$ ) and the contralaterally projecting commissural axons turn both rostrally and caudally (arrowheads, $\boldsymbol{C}, \boldsymbol{D}) . \boldsymbol{E}$, Whereas $14.3 \pm 1.6 \%(n=3190$ total number of axons in 15 open book preparations) of control GFP ${ }^{+}$axons turn ipsilaterally in the intermediate spinal cord, only $2.7 \pm 0.89 \%$ of Bmprll $\Delta$ Lim-GFP $^{+}$axons ( $n=1528$ axons in 9 open book preparations) make the ipsilateral turn ( $p<3 \times 10^{-5}$ significantly different from control, Student's t test). Similarly, only $7.4 \pm 1.5 \%$ of axons overexpressing cofilin-myc $(n=2138$ axons in 9 open book preparations) make the ipsilateral turn ( $p<0.013$ significantly different from control). $\boldsymbol{F}$, We determined the number of axons in the FP that have turned either rostrally or caudally by $\mathrm{HH}$ stage 24 . The majority of control commissural axons turn rostrally $(52.4 \pm 2.8 \%$ of axons have turned rostrally and $2.4 \pm 0.4 \%$ of axons turn caudally, $n=2507$ total number axons in the FP from 15 open book preparations), whereas significantly more of the axons electroporated with either Math $1:: B m p r l l \Delta L$ Lim-GFP (48.2 $\pm 3.9 \%$ of axons turn rostrally and $10.4 \pm 0.8 \%$ of axons turn caudally, $n=1801$ axons from 9 open book preparations, $p<7 \times 10^{-10}$ different from control) or Math1::cofilin-myc ( $46.8 \pm 3.7 \%$ of axons turn rostrally and $12.3 \pm 2.2 \%$ of axons turn caudally, $n=1813$ axons from 9 open book preparations, $p<6 \times 10^{-6}$ different from control) turn caudally. Scale bar: $A, C, 30 \mu \mathrm{m} ; \boldsymbol{B}, \boldsymbol{D}, 20 \mu \mathrm{m}$.

both directly and indirectly manipulating cofilin activity (Fig. $7 E, F)$, suggesting that specific errors are being made. A second possibility is that the errors result from the accelerated axons reaching the ventral spinal cord before the relevant guidance signals are in place to guide them. However, many of the known key guidance cues, Shh (Roelink et al., 1995), Netrin1 (Kennedy et al., 1994), and Wnts (Lyuksyutova et al., 2003), are expressed in the ventral spinal cord before the commissural axons reaching the FP. Thus, the critical guidance cue in this case might be being supplied by the oncoming commissural axons from the contralateral side of the spinal cord.

The distance over which BMP signaling can control the rate of commissural axon extension also remains unclear. Our experiments strongly suggest that BMPs act locally on commissural growth cones while they are growing in the dorsal spinal cord. However, the cell bodies of the commissural neurons remain in proximity to the source of BMPs while commissural axons are extending through the transverse trajectory of the spinal cord, raising the possibility that the BMPs could also act at a distance, with the continued activation of Bmpr signaling having a more lingering effect on the regulation of axon growth. This arrangement suggests that either the commissural neurons become insensitive or neutral to BMP signals after growing away from the RP or that the BMPs continue to provide signaling information to commissural axons, either changing their responsiveness to subsequent cues, or providing additional regulation of axon extension. Additional experiments examining whether the BMPs have differential effects when specifically applied to different compartments of the neuron should be instructive.

Together, this study suggests an additional mechanism by which guidance cues can act to direct axons. Considerable work has focused on understanding how the growth cone interprets directional information; such signals are thought to induce the local rearrangement of the actin cytoskeleton necessary to reorient the growth cone (Dickson, 2002). However, guidance factors, such as the BMPs, may also control the rate at which actin polymerizes in the growth cone to regulate the speed of growth cone extension. The rate of axon growth then determines the response of axons to subsequent guidance cues encountered along their route. In our system, the rate of axon outgrowth is "set" by guidance cues encountered earlier en route. Thus, a guidance decision could consist of two components: (1) orientation information and (2) rate information to ensure that the growth cone reaches directional signals either at the "right" developmental time or at the "right" speed to correctly interpret subsequent information, 
in an analogous manner to a car requiring a particular speed to navigate a curve in the road.

\section{Identification of an intrinsic regulator of the rate of axon outgrowth}

In summary, we propose that the balance of Limk1/cofilin activity acts within a neuron to regulate the speed of axon growth. Previous studies have shown that perturbing the levels of Limk1 in neurons can inhibit neurite growth in vitro (Endo et al., 2003). Here, we show that, by subtly modulating Limk1 activity using BMP signaling, it is possible to "tip" the balance of Limk1/cofilin activity toward promoting axonal growth. Thus, these studies also identify a cell-intrinsic way in which axon outgrowth can be accelerated in vivo. Although many extrinsic factors that promote axon outgrowth have been identified, intrinsically acting factors have been less commonly described. An understanding of how to overcome the intrinsic mechanisms by which axon growth is inhibited in vivo is likely to be important for future regenerative strategies.

\section{References}

Agnew BJ, Minamide LS, Bamburg JR (1995) Reactivation of phosphorylated actin depolymerizing factor and identification of the regulatory site. J Biol Chem 270:17582-17587.

Arber S, Barbayannis FA, Hanser H, Schneider C, Stanyon CA, Bernard O, Caroni P (1998) Regulation of actin dynamics through phosphorylation of cofilin by LIM-kinase. Nature 393:805-809.

Augsburger A, Schuchardt A, Hoskins S, Dodd J, Butler S (1999) BMPs as mediators of roof plate repulsion of commissural neurons. Neuron 24:127-141.

Birgbauer E, Solomon F (1989) A marginal band-associated protein has properties of both microtubule- and microfilament-associated proteins. J Cell Biol 109:1609-1620.

Bovolenta P, Dodd J (1990) Guidance of commissural growth cones at the floor plate in embryonic rat spinal cord. Development 109:435-447.

Bovolenta P, Dodd J (1991) Perturbation of neuronal differentiation and axon guidance in the spinal cord of mouse embryos lacking a floor plate: analysis of Danforth's short-tail mutation. Development 113:625-639.

Butler SJ, Dodd J (2003) A role for BMP heterodimers in roof platemediated repulsion of commissural axons. Neuron 38:389-401.

Butler SJ, Tear G (2007) Getting axons onto the right path: the role of transcription factors in axon guidance. Development 134:439-448.

Charron F, Tessier-Lavigne M (2005) Novel brain wiring functions for classical morphogens: a role as graded positional cues in axon guidance. Development 132:2251-2262.

Charron F, Stein E, Jeong J, McMahon AP, Tessier-Lavigne M (2003) The morphogen sonic hedgehog is an axonal chemoattractant that collaborates with netrin-1 in midline axon guidance. Cell 113:11-23.

Dickson BJ (2002) Molecular mechanisms of axon guidance. Science 298:1959-1964.

Dodd J, Jessell TM (1988) Axon guidance and the patterning of neuronal projections in vertebrates. Science 242:692-699.

Dodd J, Morton SB, Karagogeos D, Yamamoto M, Jessell TM (1988) Spatial regulation of axonal glycoprotein expression on subsets of embryonic spinal neurons. Neuron 1:105-116.

Endo M, Ohashi K, Sasaki Y, Goshima Y, Niwa R, Uemura T, Mizuno K (2003) Control of growth cone motility and morphology by LIM kinase and Slingshot via phosphorylation and dephosphorylation of cofilin. J Neurosci 23:2527-2537.

Endo M, Ohashi K, Mizuno K (2007) LIM kinase and slingshot are critical for neurite extension. J Biol Chem 282:13692-13702.

Evan GI, Lewis GK, Ramsay G, Bishop JM (1985) Isolation of monoclonal antibodies specific for human c-myc proto-oncogene product. Mol Cell Biol 5:3610-3616.

Foletta VC, Lim MA, Soosairajah J, Kelly AP, Stanley EG, Shannon M, He W, Das S, Massague J, Bernard O (2003) Direct signaling by the BMP type II receptor via the cytoskeletal regulator LIMK1. J Cell Biol [Erratum (2003) 163:421; Soosairaiah Juliana corrected to Soosairajah Juliana] 162:1089-1098.
Gehler S, Shaw AE, Sarmiere PD, Bamburg JR, Letourneau PC (2004) Brain-derived neurotrophic factor regulation of retinal growth cone filopodial dynamics is mediated through actin depolymerizing factor/ cofilin. J Neurosci 24:10741-10749.

Geisert EE Jr, Frankfurter A (1989) The neuronal response to injury as visualized by immunostaining of class III beta-tubulin in the rat. Neurosci Lett 102:137-141.

Hamburger V, Hamilton HL (1992) A series of normal stages in the development of the chick embryo. 1951. Dev Dyn 195:231-272.

Helms AW, Abney AL, Ben-Arie N, Zoghbi HY, Johnson JE (2000) Autoregulation and multiple enhancers control Math1 expression in the developing nervous system. Development 127:1185-1196.

Hogan BL (1996) Bone morphogenetic proteins: multifunctional regulators of vertebrate development. Genes Dev 10:1580-1594.

Hsieh SH, Ferraro GB, Fournier AE (2006) Myelin-associated inhibitors regulate cofilin phosphorylation and neuronal inhibition through LIM kinase and Slingshot phosphatase. J Neurosci 26:1006-1015.

Imondi R, Jevince AR, Helms AW, Johnson JE, Kaprielian Z (2007) Misexpression of $\mathrm{L} 1$ on pre-crossing spinal commissural axons disrupts pathfinding at the ventral midline. Mol Cell Neurosci 36:462-471.

Irving C, Malhas A, Guthrie S, Mason I (2002) Establishing the trochlear motor axon trajectory: role of the isthmic organiser and Fgf8. Development 129:5389-5398.

Kennedy TE, Serafini T, de la Torre JR, Tessier-Lavigne M (1994) Netrins are diffusible chemotropic factors for commissural axons in the embryonic spinal cord. Cell 78:425-435.

Lee KJ, Mendelsohn M, Jessell TM (1998) Neuronal patterning by BMPs: a requirement for GDF7 in the generation of a discrete class of commissural interneurons in the mouse spinal cord. Genes Dev 12:3394-3407.

Lee-Hoeflich ST, Causing CG, Podkowa M, Zhao X, Wrana JL, Attisano L (2004) Activation of LIMK1 by binding to the BMP receptor, BMPRII, regulates BMP-dependent dendritogenesis. EMBO J 23:4792-4801.

Liem KF Jr, Tremml G, Jessell TM (1997) A role for the roof plate and its resident TGFbeta-related proteins in neuronal patterning in the dorsal spinal cord. Cell 91:127-138.

Lyuksyutova AI, Lu CC, Milanesio N, King LA, Guo N, Wang Y, Nathans J, Tessier-Lavigne M, Zou Y (2003) Anterior-posterior guidance of commissural axons by Wnt-frizzled signaling. Science 302:1984-1988.

Marsick BM, Flynn KC, Santiago-Medina M, Bamburg JR, Letourneau PC (2010) Activation of ADF/cofilin mediates attractive growth cone turning toward nerve growth factor and netrin-1. Dev Neurobiol 70:565-588.

Meberg PJ, Bamburg JR (2000) Increase in neurite outgrowth mediated by overexpression of actin depolymerizing factor. J Neurosci 20:2459-2469.

Meng Y, Zhang Y, Tregoubov V, Janus C, Cruz L, Jackson M, Lu WY, MacDonald JF, Wang JY, Falls DL, Jia Z (2002) Abnormal spine morphology and enhanced LTP in LIMK-1 knockout mice. Neuron 35:121-133.

Mortimer D, Pujic Z, Vaughan T, Thompson AW, Feldner J, Vetter I, Goodhill GJ (2010) Axon guidance by growth-rate modulation. Proc Natl Acad Sci U S A 107:5202-5207.

Piper M, Anderson R, Dwivedy A, Weinl C, van Horck F, Leung KM, Cogill E, Holt C (2006) Signaling mechanisms underlying Slit2-induced collapse of Xenopus retinal growth cones. Neuron 49:215-228.

Placzek M, Tessier-Lavigne M, Jessell T, Dodd J (1990) Orientation of commissural axons in vitro in response to a floor plate-derived chemoattractant. Development 110:19-30.

Roelink H, Porter JA, Chiang C, Tanabe Y, Chang DT, Beachy PA, Jessell TM (1995) Floor plate and motor neuron induction by different concentrations of the amino-terminal cleavage product of sonic hedgehog autoproteolysis. Cell 81:445-455.

Rosenzweig BL, Imamura T, Okadome T, Cox GN, Yamashita H, ten Dijke P, Heldin CH, Miyazono K (1995) Cloning and characterization of a human type II receptor for bone morphogenetic proteins. Proc Natl Acad Sci U S A 92:7632-7636.

Ruegg MA, Stoeckli ET, Kuhn TB, Heller M, Zuellig R, Sonderegger P (1989) Purification of axonin-1, a protein that is secreted from axons during neurogenesis. EMBO J 8:55-63.

Sarmiere PD, Bamburg JR (2004) Regulation of the neuronal actin cytoskeleton by ADF/cofilin. J Neurobiol 58:103-117.

Shirasaki R, Lewcock JW, Lettieri K, Pfaff SL (2006) FGF as a target-derived chemoattractant for developing motor axons genetically programmed by the LIM code. Neuron 50:841-853. 
Strochlic L, Dwivedy A, van Horck FP, Falk J, Holt CE (2008) A role for S1P signalling in axon guidance in the Xenopus visual system. Development 135:333-342.

Tessier-Lavigne M, Placzek M, Lumsden AG, Dodd J, Jessell TM (1988) Chemotropic guidance of developing axons in the mammalian central nervous system. Nature 336:775-778.

Timmer J, Johnson J, Niswander L (2001) The use of in ovo electroporation for the rapid analysis of neural-specific murine enhancers. Genesis 29:123-132.

Tursun B, Schlüter A, Peters MA, Viehweger B, Ostendorff HP, Soosairajah J, Drung A, Bossenz M, Johnsen SA, Schweizer M, Bernard O, Bach I (2005) The ubiquitin ligase Rnf6 regulates local LIM kinase 1 levels in axonal growth cones. Genes Dev 19:2307-2319.

Wen Z, Han L, Bamburg JR, Shim S, Ming GL, Zheng JQ (2007) BMP gra- dients steer nerve growth cones by a balancing act of LIM kinase and Slingshot phosphatase on ADF/cofilin. J Cell Biol 178:107-119.

Wilson SI, Shafer B, Lee KJ, Dodd J (2008) A molecular program for contralateral trajectory: Rig-1 control by LIM homeodomain transcription factors. Neuron 59:413-424.

Wine-Lee L, Ahn KJ, Richardson RD, Mishina Y, Lyons KM, Crenshaw EB 3rd (2004) Signaling through BMP type 1 receptors is required for development of interneuron cell types in the dorsal spinal cord. Development 131:5393-5403.

Yamauchi K, Phan KD, Butler SJ (2008) BMP type I receptor complexes have distinct activities mediating cell fate and axon guidance decisions. Development 135:1119-1128.

Zou Y, Lyuksyutova AI (2007) Morphogens as conserved axon guidance cues. Curr Opin Neurobiol 17:22-28. 